\title{
Many candidates for a single chair: a critical review of the genetic determinant of flat fruit shape trait in peach (Prunus persica L. Batsch)
}

\author{
Marco Cirilli $^{1}$ (D) Laura Rossini ${ }^{1}$
}

Received: 7 January 2021 / Revised: 17 March 2021 / Accepted: 9 June 2021 / Published online: 19 June 2021

(c) The Author(s) 2021

\begin{abstract}
Known since ancient times, flat-shaped peach (Prunus persica $\mathrm{L}$. Batsch) fruits have aroused considerable research interest. Breeding activities for this trait have expanded since the 1980s of the last century in several countries, in parallel with research into its genetic basis. Following the need for molecular markers to assist selection, linkage mapping studies have positioned the flat shape $S$ locus at the end of chromosome 6. Recently, a series of independent studies focused on detailed characterization of the genomic region harbouring the $S$ locus and different hypotheses about the candidate gene or variant were proposed: from a constitutively activated cell death 1 protein $(P p C A D 1)$ to a Brassinosteroid insensitive 1-associated receptor kinase 1 group (PpLRR-RLK) until the discovery of large structural variant (a chromosomal inversion of about $1.7 \mathrm{Mb}$ ) putatively affecting an OVATE Family Protein (PpOFP1) and a homolog of sucrose non-fermenting 1-related kinase (PpSNF4), respectively, located at the proximal and distal breakpoints. This short review revises historical studies and recent literature, integrating reanalysis of available genetic and genomic data, to provide a critical overview of the topic and highlight avenues for further research.
\end{abstract}

Keywords Fruit quality $\cdot$ Shape $\cdot$ Breeding $\cdot$ Variant $\cdot$ Locus $\cdot$ Gene

\section{A short breeding history of flat fruit trait}

Reported in the Middle Age book Luo Yang Hua Mu Ji of the author Zhou Shi Hou (1081 AD), the flat shape of peach fruits ('Pan Tao' in Chinese) has long been a botanical interest in Occidental countries (Huang et al. 2008). This type of peach was first introduced by the Chinese in Australia (at the beginning of the nineteenth century) and from there to the USA (in 1869), with the name of 'Australian Saucer'. Early breeding activities used this variety and derived materials (such as 'Peento', 'Angel' and 'Jewel') as a source of low-chill requirement for adaptation to the subtropical climate (Okie et al. 2008). Around 1950, flat peaches were introduced in Sicily (Italy), where they are still cultivated

Communicated by S.C. González-Martínez

Marco Cirilli

marco.cirilli@unimi.it

$\triangle$ Laura Rossini

laura.rossini@unimi.it

1 Department of Agricultural and Environmental Sciences (DISAA), University of Milan, Via Celoria 2, Milan, Italy in some niche areas and known with the name of 'Tabacchiere'. The variety 'Stark Saturn' (1985), bred as NJF-2 by L.F. Hough at Rutgers University (New Brunswick, N.J.) and commercialized by Starks Nursery, was probably the first flat peach destined for fresh market consumption (also known as saucer or donut) (Byrne 2005). Cold hardiness was the major feature of this variety, which allows the expansion of cultivation environments for this varietal type. Breeding programmes for flat peaches only started at the end of 1980s, mostly resorting on 'Stark Saturn', particularly in France (noteworthy releases were 'Platina' and 'Mesembrine') (Monet et al. 1985; Monet 1986; Pascal and Monteux-Caillet 1998), Italy ('UFO' series) (Nicotra and Conte 2003), China ('Ruipan' series) (Ma et al. 2015), and USA (several public or private programmes) (Sherman and Lyrene 2001). Since early 2000s, Spain placed special emphasis on flat breeding, as a support for a remarkable expansion of the market segment for this peach type in some countries, with some programmes still ongoing (Batlle et al. 2012). 


\section{Genetic characterization of the $S$ locus}

The first genetic characterization dates back to Lesley (1940), who described the inheritance of the flat (saucer) trait as Mendelian, monogenic and dominant over round shape $(S / s)$. The $S$ locus was mapped for the first time to the bottom of linkage group 6 (Dirlewanger et al. 1998). Other attempts to find molecular markers associated to the $S$ locus resulted in the identification of the RAPD marker OPP09950 (Guo et al. 2002). A second-generation map integrating SSR markers was then provided by Dirlewanger et al. (2006). In this seminal work, an $F_{2}$ segregating progeny of about 200 individuals was obtained from self-pollination of a heterozygous flat $\mathrm{F}_{2}$ seedling of 'Ferjalou Jalousia' (a flat peach) x 'Fantasia' (a round nectarine) (hereafter JxF progeny). Based on SSR, AFLP and morphological markers, the $S$ locus co-segregated with MA014a and MA040a (SSR), and pCC-GAA/c (AFLP) at $72.6 \mathrm{cM}$. Interestingly, three phenotypes segregated in agreement to the Mendelian 1:2:1 ratio: round, flat and flat aborting fruit (named Af). This last phenotype is characterized by malformed fruits aborting during the S1 stage of development (the first exponential growth phase of rapid increase in cell division rate) and also displaying early cracking on the pistilar side (as further described by Lambert et al. 2009). The segregation pattern of $S$ and $A f$ traits suggested the existence of a single variant controlling both traits (i.e. $s s$, round, $S s$, flat and $S S$ flat aborting) or, alternatively, two variants in tight linkage (i.e. the dominant $S$ allele linked to the recessive af allele causing the aborting fruit phenotype). SSR marker MA014a (although not perfectly associated to the phenotype) has been used in marker-assisted selection of flat-fruited cultivars, allowing the reduction of breeding costs and time (Lambert et al. 2009).

An independent study by Picañol et al. (2013), using a small segregating progeny of 73 individuals from the cross 'UFO-3' (flat) x 'Sweet Cap' (flat), confirmed previous mapping results without restricting the associated interval. Authors also tested the performance of some SSR markers at locus $S$ in their germplasm, finding best co-segregation for UDP98-412 (Pp06:26,617,798-26,617,926) and MA040a.

\section{Searching for the candidate gene variant}

Following the release of the peach genome sequence (Verde et al. 2013), building upon the chromosomal position of the $S$ locus (spanning about $3 \mathrm{Mb}$ ) and the strong association of the flat trait with some SSR markers, different research groups moved from classical genetics to genome-wide approaches in search of the causal gene(s) and/or causal variant(s) (Micheletti et al. 2015). This approach led to a series of publications identifying at least four different candidate genes for the $S$ trait: Prupe.6G292200, having homology with a constitutively activated cell death 1 protein (hereafter PpCAD1) (Cao et al. 2016); Prupe.6G281100, similar to a leucine-rich repeat receptor-like kinase orthologous to the Brassinosteroid insensitive 1-associated receptor kinase 1 group (PpLRR-RLK) (López-Girona et al. 2017); Prupe.6G290900 encoding a putative OVATE Family Protein (PpOFPl) (Zhou et al. 2021; Guo et al. 2020; Guan et al. 2021); and Prupe.6G323700, homologous of sucrose non-fermenting 1-related kinase, (PpSnRK1 $\beta \gamma / P p S N F 4)$ (Guo et al. 2021). Before discussing the rationale behind each candidate gene claim in the abovementioned studies, we first attempt to improve the resolution of the mapped interval at the $S$ locus. For this purpose, we use data from $141 \mathrm{~F}_{2} \mathrm{JxF}$ progenies genotyped with the $9 \mathrm{~K}$ SNP peach array (data available from Hernández Mora et al. 2017). This dataset includes the same individuals used for the construction of the third-generation map of the $S$ locus, excluding the aborting type, and locating the gene within a region of 2,595,690 bp (Lambert et al. 2016). The position of co-segregating marker MA040a (Pp06:26,722,150-26,722,384) and the nearby MA014a (Pp06:27,186,703 - 27,186,851) and AMPA121 (Pp06:28,325,430-28,325,565) were also incorporated. Linkage analysis allows restriction of the associated interval to the region spanning 2,539,266 bp comprised between SNP_IGA_690016 and SNP_IGA_699045 (corresponding to the physical region $26,778,808-29,318,074 \mathrm{bp}$ ). This reconstruction of recombination events is also in agreement with MA040a and $S$ positions in the map shown by Lambert et al. (2016). Such interval de facto excludes the candidate variant within PpLRR-RLK (a deletion spanning about $10 \mathrm{~Kb}$ upstream of the start codon and $693 \mathrm{bp}$ downstream), proposed by López-Girona et al. (2017) based on co-segregation with flat trait in a panel of 246 cultivars. Notably, at least 3 recombination events support the exclusion of this variant as the determinant of flat trait (Fig. 1 and Supplemental File 1). Interestingly, López-Girona et al. (2017) also described a round natural somatic revertant of the flat variety 'UFO-4', although they failed to obtain the sequence of the mutated flat PpLRR-RLK allele. Another round bud sport revertant was also described by Tan et al. (2019) in the flat cultivar 'Zhaoyue'. Comparison of whole-genome re-sequencing (WGRS) data of LII mesocarp layers of both fruit types revealed a sharp reduction of the heterozygosity levels in the round fruit mutant, starting from about $22 \mathrm{Mbp}$ of $\mathrm{Chr}$ 6 until the telomere. The flat to round mutation seems probably caused by the replacement of the chromosomal region bearing the flat allele with the round one, perhaps as a result of mitotic recombination (Foster and Aranzana 2018). Anyway, the size of the replaced region is too wide to be useful for restriction of the $S$ locus. 


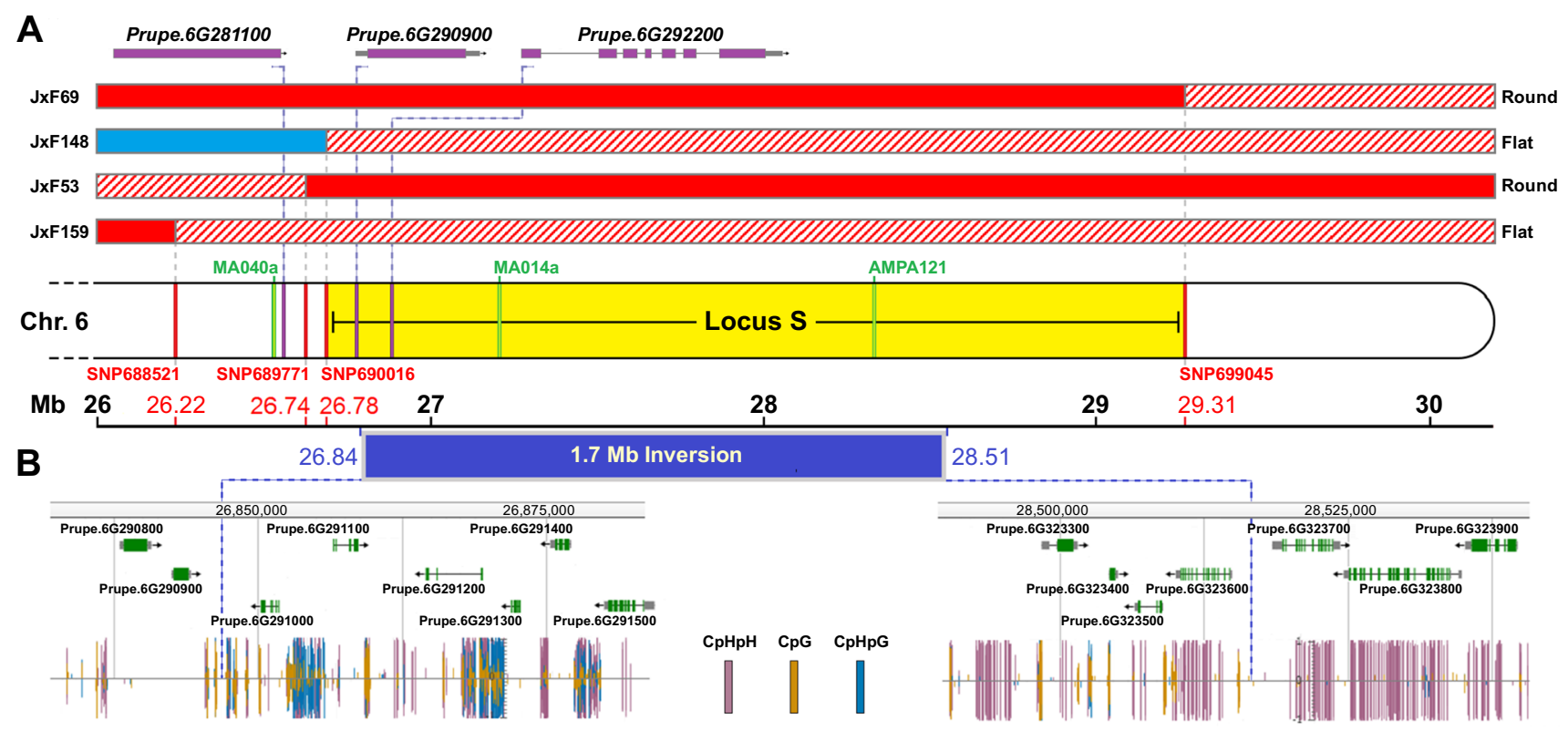

Fig. 1 A Locus $S$ SNP map obtained from segregation of $141 \mathrm{~F}_{2} \mathrm{JxF}$ seedlings. Recombinant individuals \#69, \#148, \#53 and \#159 delimited the interval to about $2.5 \mathrm{Mb}$ at the distal end of chromosome 6. The position of the candidate genes Prupe.6G281100 (PpLRRRLK), Prupe.6G290900 (PpOFPl) and Prupe.6G292200 (PpCAD1) are shown, along with the position of $1.7 \mathrm{Mb}$ insertions in the flatassociated haplotype. The two additional large inversions reported by Zhou et al. (2021) of 781,720 and 2,125,678 bp, respectively,

In contrast to $P p L R R-R L K$ and its deletion, a SNP variant within PpCADI falls within the restricted interval shown in Fig. 1. This candidate was proposed after identifying a major association peak in a panel of 129 accessions, consisting of an $\mathrm{A} / \mathrm{T}$ nucleotide polymorphism in the fifth intron of the gene (Cao et al. 2016). A different expression level between flat and round genotypes was shown as support of the involvement of this gene, although assayed tissues were sampled from mature fruits, a stage where morphological differences in peach shape are already developed. The A allele showed a perfect co-segregation with the flat trait in a panel of 474 peach accessions and wild relatives. As also criticized by López-Girona et al. (2017), the flat-associated A allele was homozygous in one flat cultivar, in apparent disagreement with the incomplete dominance model of a single variant responsible for phenotypic effects of varying severity (i.e. homozygous A should have an aborting phenotype). In fact, such allelic configuration is still compatible with the hypothesis of two tightly linked genes, although Cao et al. (2016) did not discuss the possibility of a linkagebreak in this homozygous flat cultivar. In a subsequent paper, the same group (Guo et al. 2018) reported some inconsistencies in 18 flat cultivars present in their germplasm collection when genotyped with the PpLRR-RLK variant of López-Girona et al. (2017) — this lack of co-segregation between 27,776,947 - 28,558,667 and 28,096,494 - 30,222,172 are also shown. Colours of the recombinant blocks indicate haplotypes derived from the 'Fantasia' (red), 'Ferjalou Jalousia' (light blue) and heterozygous (red side bars). The map position (in cM) of SSR markers from Dirlewanger et al. (2006) is also shown. B Relative percentage of putatively methylated sites around the inverted sites as observed in 'Lovell' leaves samples: $\mathrm{CpG}$ (orange), $\mathrm{CpHpG}$ (blue) and $\mathrm{CpHpH}$ (violet), according to Plant Methylome DB nomenclature

with the phenotype is consistent with this variant falling out of the mapping interval, as previously shown in Fig. 1. Curiously, based on RNAseq and gene expression data, Guo et al. (2018) partially retracted the involvement of CAD1 in consideration of the lack of significant differences in its relative expression levels between flat and round fruits during early fruit development. In this case, developing a self-pollinated progeny from the homozygous A flat cultivar could better clarify the actual involvement of the CAD1 variant (i.e. to be valid the 2 linked genes hypothesis, seedlings should have been all flats).

Recently, another candidate variant has been proposed, which falls within the restricted interval (Fig. 1) and consists of a large chromosomal inversion of about $1.67 \mathrm{Mb}$ (hereafter 1.7-INV) in flat cultivars (compared to the reference genome of the round fruit accession 'Lovell'). This rearrangement was reported by three closely published studies (Zhou et al. 2021; Guo et al. 2020; Guan et al. 2021) as putatively affecting candidate gene Prupe.6G290900, encoding a putative OVATE Family Protein. Chronologically, this variant has been first reported by Zhou et al. (2021) based on PacBio sequencing of the flat cultivar '124 Pan'. Proximal (PB) and distal (DB) breakpoints of 1.7-INV are located at positions $26,847,156$ and 28,516,622, respectively. The two haplotypes without (H1) or with the inversion (H2) 
perfectly co-segregate in a wide panel of more than 700 accessions from peach and other related species, being $\mathrm{H} 1 \mathrm{H} 2$ flat, $\mathrm{H} 1 \mathrm{H} 1$ round and $\mathrm{H} 2 \mathrm{H} 2$ flat aborting. Haplotypes were obtained by analysing available Illumina re-sequencing data (although several libraries are characterized by a low sequencing depth). Noteworthy, the 1.7-INV was also found in two $P$. ferganensis accessions with flat and aborting phenotypes $(\mathrm{H} 1 \mathrm{H} 2$ and $\mathrm{H} 2 \mathrm{H} 2$, respectively). However, among the various Sequence Read Archive datasets screened by the authors, we noticed a P. ferganensis accession with an apparent flat fruit name ('Pan Tao Weihai') but not considered in their analysis. We analysed WGRS data of this accession by adopting the same approach described by Zhou et al. (2021), failing to detect the presence of the 1.7-INV variant (Fig. 2). Notably, the flat and probably aborting phenotype of 'Pan Tao Weihai' has been confirmed by Dr. Hua Xie (Beijing Academy of Agricultural and Forestry Sciences, personal communication) co-author in the work releasing the dataset (BioSample SAMN04447981, Yu et al. 2018). Therefore, at least more than one allele would appear to confer the $S$ trait in peach. Other aspects of the work remain unclear. Firstly, PacBio sequencing showed the presence of two additional large inversions, both overlapping with the DB border of 1.7-INV (Fig. 1). Such observation was not further addressed raising the question of the actual structure of the chromosomal rearrangement of distal end of $\mathrm{Chr}$ 6. However, recombination events detected in the JxF SNP map from about $29 \mathrm{Mb}$ onwards suggest that at least the most distal inversion may be due to an assembling error (Supplemental File 1): verification of the actual presence of the two additional inversions (as well as the haplotype they may belong to) would be particularly relevant in light of the recombination event occurring at marker AMPA121 reported in the map by Dirlewanger et al. (2006). This SSR is localized within the $1.7 \mathrm{Mb}$-INV region $(28,325,430$ $-28,325,565)$ in contrast with the expected suppression of meiotic recombination induced by a paracentric chromosomal inversion in heterozygosis (Zapata et al. 2016). In the case of the $S$ locus, the inversion should cause an absence of recombination between heterozygous alleles. While a genotyping error in $\mathrm{JxF}$ progeny is the most likely explanation, other rare events could not be excluded a priori (i.e. a chromosomal rearrangement or double cross-over) (Kirkpatrick 2010). Following Zhou et al. (2021), two other studies also
Fig. 2 Reads alignment in a $250 \mathrm{bp}$ window around the proximal (PB) and distal (DB) breakpoints in the flat (probably aborting) $P$. ferganensis 'Pan Tao Weihai' accession. For this analysis, sequences of round $(\mathrm{H} 1)$ and flat $(\mathrm{H} 2)$ alleles were used as query. At the PB border, 'Pan Tao Weihai' sequence reads span the round $\mathrm{H} 1$ allele (violet) but not the flat $\mathrm{H} 2$ allele (split reads in blue), clearly indicating the absence of a breakpoint. In contrast, the alignment at the DB border is unclear in either $\mathrm{H} 1$ or $\mathrm{H} 2$ alleles

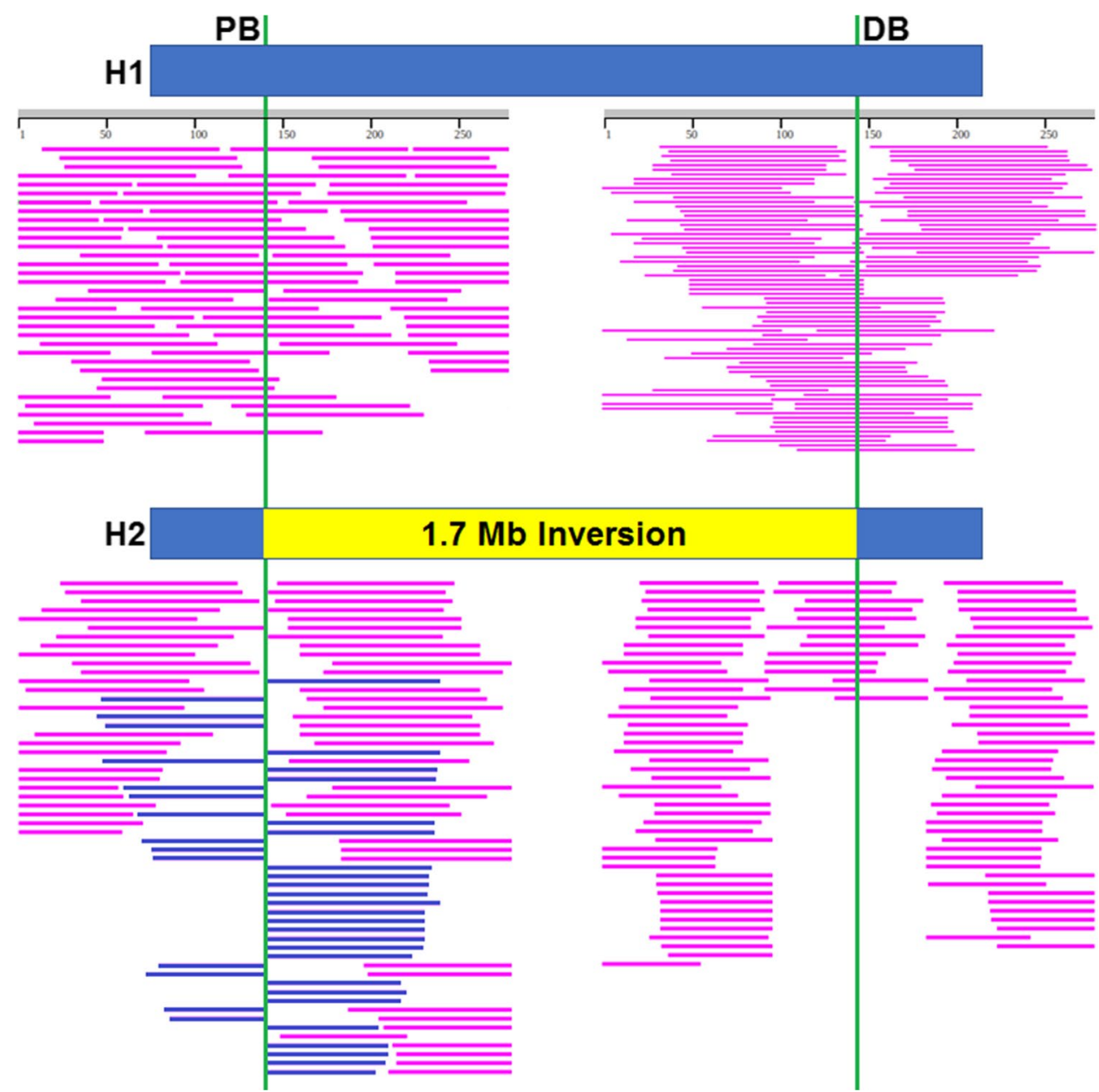


reported the identification (and validation in their respective germplasm collections) of the 1.7-INV through Pac-Bio sequencing of 'Xinjiang Pan Tao \#2', an aborting-type $P$. ferganensis accession (Guo et al. 2020), and 'Rui You Pan 1', a flat peach cultivar (Guan et al. 2021). Of particular importance, Guan et al. (2021) apparently reported a unique large chromosomal rearrangement at the end of chromosome 6 (i.e. the 1.7-INV) in comparison to Zhou et al. (2021), probably due to the adoption of a more stringent cut-off for structural variant identification. Authors also speculated about the hypothesis of recombination suppression induced by 1.7-INV as supported by high linkage disequilibrium (LD) levels between SNPs positioned within or adjacent to the inversion in the flat-fruit accessions as compared to the round-fruit ones.

Zhou et al. (2021), Guo et al. (2020) and Guan et al. (2021) concentrated their efforts on the proximal breakpoint downstream of Prupe.6G290900 (curiously renamed PpOFP2 by Guan et al. (2021) and hereafter indicated only as $P p O F P 1)$. This gene is by far the most promising candidate, considering the well-established role of OFP family members in tomato, where they synergistically regulate fruit shape during early organ development by primarily modulating cell division patterns through the OFP-TRM module (Liu et al. 2002). In particular, a deletion in the upstream regulatory region of SIOFP2O underlying a suppressor of ovate (sov1) locus is responsible for the elongated shape in the OVATE null mutation background (Wu et al. 2018). The over-expression (OE) of SlOFP20 causes the production of rounder fruits in 'Yellow Pear' (Wu et al. 2018) and flat/ oblate in 'Alisa Craig' (albeit, only one line is shown) (Zhou et al. 2019). Consistent with this, Zhou et al. (2021), Guo et al. (2020) and Guan et al. (2021) found a significant upregulation of PpOFPl in flat fruits at early stages of development comparing several flat and round cultivars. Indeed, shape differences originate during the $\mathrm{S} 1$ phase of rapid cell division and derive from a reduced cell number along the vertical axis in flat fruits. An additional layer of validation was provided by tomato transformation: transgenic 'MicroTom' lines overexpressing peach PpOFPl showed a flattened fruit shape (along with some developmental aberrations) due to cell number reduction along the vertical axis, further supporting a conserved function of this gene (Guo et al. 2020; Guan et al. 2021, probably independent experiments). By the way, developmental aberrations in tomato fruits may suggest deleterious effects associated to elevated expression of this gene; curiously, this actually occurs in flat aborting peach fruits, in which $P p O F P 1$ is expressed at higher level compared to the flat ones, as observed by Guo et al. (2020). However, authors did not advance such a hypothesis, leaving uncommented this result.

The putative effect of 1.7-INV at the DB breakpoint has been less thoroughly addressed by Zhou et al. (2021), although RNAseq data indicated some promising difference in the expression of Prupe.6G323700 gene, encoding a homologous of sucrose non-fermenting 1-related kinase. Guo et al. (2020) observed a higher expression level in round versus flat. However, comparing the divergent expression pattern of Prupe.6G323700 and PpOFP1, authors questionably concluded that given the dominant inheritance of flat shape, the candidate gene with the higher expression levels (i.e. $P p O F P 1$ ) was the more likely responsible for the flat phenotype. Guan et al. (2021) analysed expression pattern of Prupe.6G323700 (named in this work PpSNF4) in 136 accessions, confirming significantly higher transcript levels in the round versus flat group, while some accessions appeared to deviate from this general trend. This was probably the reason inducing authors to speculate about a mechanism of lethal dosage of $P$ POFPl expression as the cause of flat fruit abortion, even if they apparently do not include any aborting-type accessions in their analyses. Later on, Guo et al. (2021) attempted to further characterize the role of Prupe.6G323700 (renamed PpSnRK $\beta 1$ and hereafter indicated only as PpSNF4). Apart from an allelic-dependent expression (almost undetectable in the flat aborting genotype and highest in the round one), stable (in tomato) and transient (in peach fruit sections) over-expression of this gene increased both starch and sugar content, indicating a putative role of in carbohydrate metabolism. Dual-Luciferase assay of the PpSNF4 promoter rearrangement caused by 1.7-INV showed a reduced activity in a 'flat' versus 'round' genotype. Collectively, these findings lead authors to attribute the flat aborting phenotype to a down-regulation of this gene. Nevertheless, authors only generated transgenic 'Micro-Tom' tomato OE lines rather than trying to silence the tomato ortholog of PpSNF4 (to test for possible fruit abortion or other lethal effects on development), thus failing to provide convincing evidence in support of their hypothesis.

\section{Discussions and concluding remarks}

Molecular characterization of the locus conferring a flat fruit shape in peach has aroused a growing interest from various research groups. In this concise review, we critically reanalyse the relevant literature on the topic with the aim of clarifying and identifying the main outcomes of previous research and outline some aspects to be further addressed in the future. Collectively, the $1.7 \mathrm{Mb}$ inversion recently uncovered by the investigations of Zhou et al. (2021), Guo et al. (2020) and Guan et al. (2021) seems to be the strongest candidate causal factor behind locus $S$, as supported by its perfect co-segregation in a very large number of peach and related accessions. This is also in agreement with suppressed recombination in the locus $S$ genomic region in the $\mathrm{JxF}$ progeny. 
For breeding purposes, the identification of 1.7-INV will clearly improve the reliability of marker-assisted selection of the flat trait, although the performances of previously developed markers have been already proven to be more than adequate for the scope (Lambert et al. 2009; Lambert et al. 2016; Zhang et al. 2018).

At the scientific level, this finding opens a series of interesting questions. Indeed, chromosomal inversions are considered important for many evolutionary processes, although very few examples of inversion-associated functional variants and traits have been described in plants (Hoffmann and Rieseberg 2008). Primarily, we still need to understand what gene(s) or genetic variant(s) inside or at the breakpoints of the 1.7-INV is important and the exact molecular mechanism(s) leading to the associated phenotypes (round, flat and aborting fruit): the variant(s) could be (i) the breakpoints regions themselves, disrupting gene promoter regions of PpOFP1 and/or PpSNF4 through nucleotide mutations adjacent to breakpoints, or reorganization of regulatory domains (i.e. maintenance of linkage with short- or longrange cis or trans regulatory elements), or a local modification of the epigenetic environment (chromatin remodelling, histone modification or DNA methylation) or (ii) the regions between the breakpoints, linked by recombination suppression (i.e. the $1.7 \mathrm{Mb}$ inversions contain many functional variants potentially affecting multiple downstream phenotypes) (Said et al. 2018). In case of inversion-induced epigenetic changes, histone and DNA methylation studies have been already performed in peach (Leida et al. 2012; Zhu et al., 2020), making feasible the addition of this level of knowledge. For example, different distributions of $\mathrm{CpG}$, $\mathrm{CpHpG}$ and $\mathrm{CpHpH}$ islands may exist across the two breakpoint borders as found for leaf tissues based on peach methylome data available at Plant Methylome DB website (http:// epigenome.genetics.uga.edu/PlantMethylome/) (Fig. 1). In contrast, resolving the hypothesis of an independent effect of different variants within an inversion locus is difficult to achieve through fine-mapping or genome-wide association approaches, due to recombination suppression between heterokaryotypes or elevated linkage disequilibrium. The works of Zhou et al. (2021), Guo et al. (2020), Guan et al. (2021) and Guo et al. (2021) all focused on the inversion breakpoints (although without clarifying genetic variant(s)), mainly supported by transcriptional evidences. Whether $P p O F P 1$ and/or PpSNF4 genes underlie the flat shape and the flat abortive traits, respectively, or $P p O F P 1$ acts in a dosage-dependent manner to control both, or one or other genes are in close linkage cannot be conclusively demonstrated by transcriptional regulation evidence. In spite of this, $P p O F P 1$ is by far the most promising candidate, at least for the flat shape of fruits, mainly supported by tomato genetic engineering and the extensive studies performed on OVATE family members in this model species for fleshy fruits. The difficulty of engineering peach, a species recalcitrant to in vitro regeneration, is still representing a considerable obstacle for such molecular genetic studies. Ideally, site-specific recombination systems, including CRISPR/Cas, are powerful tools for chromosomal engineering with the potential to invert DNA regions and modify local recombination patterns (Pacher et al. 2007; Schmidt et al. 2020).

Beyond the identification of the responsible variant for the flat fruit shape, previously cited Pac-Bio genome sequencing works suggest the occurrence of several chromosomal rearrangements across different accessions and the power of single molecule real-time (SMRT) approaches for their detection. Besides basic scientific research, the impact of chromosomal variations in shaping the recombination landscape and genetic variability in peach will be highly relevant for applicative breeding purposes.

Supplementary Information The online version contains supplementary material available at https://doi.org/10.1007/s11295-021-01515-w.

Funding Open access funding provided by Università degli Studi di Milano within the CRUI-CARE Agreement.

Open Access This article is licensed under a Creative Commons Attribution 4.0 International License, which permits use, sharing, adaptation, distribution and reproduction in any medium or format, as long as you give appropriate credit to the original author(s) and the source, provide a link to the Creative Commons licence, and indicate if changes were made. The images or other third party material in this article are included in the article's Creative Commons licence, unless indicated otherwise in a credit line to the material. If material is not included in the article's Creative Commons licence and your intended use is not permitted by statutory regulation or exceeds the permitted use, you will need to obtain permission directly from the copyright holder. To view a copy of this licence, visit http://creativecommons.org/licenses/by/4.0/.

\section{References}

Batlle I, Fontich C, Lozano L, Iglesias I, Reig G, Alegre S et al (2012) The peach breeding programme IRTA-ASF: aiming for high fruit quality. Acta Hortic 940:75-78

Byrne D (2005) Trends in stone fruit cultivar development. HortTechnol 15(3):494-500

Cao K, Zhou Z, Wang Q et al (2016) Genome-wide association study of 12 agronomic traits in peach. Nat Commun 7:13246

Dirlewanger E, Pronier V, Parvery C, Rothan C, Guye A, Monet R (1998) Genetic linkage map of peach [Prunus persica (L.) Batsch] using morphological and molecular markers. Theor Appl Genet 97:888-895

Dirlewanger E, Cosson P, Boudehri K, Renaud C, Capdeville G, Tauzin $Y$ et al (2006) Development of a second-generation genetic linkage map for peach [Prunus persica (L.) Batsch] and characterization of morphological traits affecting flower and fruit. Tree Genet Genomes 3:1-13

Foster TM, Aranzana MJ (2018) Attention sports fans! The far-reaching contributions of bud sport mutants to horticulture and plant biology. Hortic Res 5:44 
Guan J, Xu Y, Yu Y, Fu J, Ren F, Guo J et al. (2021) Genome structure variation analyses of peach reveal population dynamics and a 1.67 $\mathrm{Mb}$ causal inversion for fruit shape. Genome Biol 22:13

Guo J, Jiang Q, Zhang K, Zhao J (2002) Screening for the molecular marker linked to saucer gene of peach fruit shape. Acta Hortic 592:267-271

Guo J, Cao K, Li Y, Yao JL, Deng C, Wang Q et al (2018) Comparative transcriptome and microscopy analyses provide insights into flat shape formation in peach (Prunus persica). Front Plant Sci 8:2215

Guo J, Cao K, Deng C et al (2020) An integrated peach genome structural variation map uncovers genes associated with fruit traits. Genome Biol 21:258

Guo J, Cao K, Yao JL et al (2021) Reduced expression of a subunit gene of sucrose non-fermenting 1 related kinase, $P p S n R K 1 \beta \gamma$, confers flat fruit abortion in peach by regulating sugar and starch metabolism. BMC Plant Biol 21:88

Hernández Mora JR, Micheletti D, Bink M, Van de Weg E, Bassi D, Nazzicari N et al (2017) Integrated QTL detection for key breeding traits in multiple peach progenies. BMC Genomics 18(1):404

Hoffmann AA, Rieseberg LH (2008) Revisiting the impact of inversions in evolution: from population genetic markers to drivers of adaptive shifts and speciation? Annu Rev Ecol Evol Syst 39:21-42

Huang H, Cheng Z, Zhang Z, Wang Y (2008) History of cultivation and trends in China. In: Layne DR, Bassi D (eds) The peach - Botany. CAB International, Production and Uses, pp 37-70

Kirkpatrick M (2010) How and why chromosome inversions evolve. PLoS Biol 8: e1000501

Lambert P, Dirlewanger E, Laurens F (2009) La sélection assistée par marqueurs (SAM) chez les arbres fruitiers: une approche prometteuse au service de l'innovation variétale. Innovations Agronomiques 7:139-152

Lambert P, Campoy JA, Pacheco I, Mauroux J, Linge CDS, Micheletti D, Bassi D, Rossini L, Dirlewanger E, Pascal T (2016) Identifying SNP markers tightly associated with six major genes in peach [Prunus persica (L.) Batsch] using a high-density SNP array with an objective of marker-assisted selection (MAS). Tree Genet Genomes 12:1-21

Leida C, Conesa A, Llacer G, Badenes ML, Rios G (2012) Histone modifications and expression of DAM6 gene in peach are modulated during bud dormancy release in a cultivar-dependent manner. N Phytol 193:67-80

Lesley J (1940) A genetic study of saucer fruit shape and other characters in the peach. Proc Am Soc Hortic Sci 37:218-222

Liu J, Van Eck J, Cong B, Tanksley SD (2002) A new class of regulatory genes underlying the cause of pear-shaped tomato fruit. Proc Natl Acad Sci USA 99:13302-13306

López-Girona E, Zhang Y, Eduardo I et al (2017) A deletion affecting an LRR-RLK gene co-segregates with the fruit flat shape trait in peach. Sci Rep 7:6714

Ma R, Yu M, Xu J, Zhang Y, Shen Z (2015) Progress on peach breeding in China. Acta Hortic 1084:271-276

Micheletti D et al (2015) Whole-Genome analysis of diversity and SNP-major gene association in peach germplasm. PLoS ONE 10:e 0136803

Monet R, Bastard Y, Gibault B (1985) Genetic-studies on the breeding of flat peaches. Agronomie 5(8):727-731

Monet R (1986) Flat peach are new at the market. Recherche 17(179):1000-1002

Nicotra A, Conte L (2003) New fruit typology for the peach market: birth of the "Ufo" and "Ghiaccio" series. Rivista Di Frutticoltura e Di Ortofloricoltura 65:20-25
Okie WR, Bacon T, Bassi D (2008) Fresh market cultivar development. In: Layne DR, Bassi D (eds) The peach - Botany. CAB International, Production and Uses, pp 139-174

Pacher M, Schmidt-Puchta W, Puchta H (2007) Two unlinked doublestrand breaks can induce reciprocal exchanges in plant genomes via homologous recombination and nonhomologous end joining. Genetics 175:21-29

Pascal T, Monteux-Caillet R (1998) Peach breeding in France. Acta Hortic 465:117-124

Picañol R, Eduardo I, Aranzana MJ, Howad W, Batlle I, Iglesias I, Alonso JM, Arús P (2013) Combining linkage and association mapping to search for markers linked to the flat fruit character in peach. Euphytica 190:279-288

Said I, Byrne A, Serrano V, Cardeno C, Vollmers C, Corbett-Detig R (2018) Linked genetic variation and not genome structure causes widespread differential expression associated with chromosomal inversions. Proc Natl Acad Sci USA 115:5492-5497

Schmidt C, Fransz P, Rönspies M et al (2020) Changing local recombination patterns in Arabidopsis by CRISPR/Cas mediated chromosome engineering. Nat Commun 11:4418

Sherman WB, Lyrene PM (2001) 'UFO' a saucer or donut peach. J Amer Pomological Soc 55(1):2-3

Tan Q, Liu X, Gao H, Xiao W, Chen X, Fu X et al (2019) Comparison between flat and round peaches, genomic evidences of heterozygosity events. Front Plant Sci 10:592

Verde I, Abbott AG, Scalabrin S, Jung S, Shu S, Marroni F et al (2013) The high-quality draft genome of peach (Prunus persica) identifies unique patterns of genetic diversity, domestication and genome evolution. Nat Genet 45:487-494

Wu S, Zhang B, Keyhaninejad N, Rodriguez GR, Kim HJ, Chakrabarti $\mathrm{M}$ et al (2018) A common genetic mechanism underlies morphological diversity in fruits and other plant organs. Nat Commun 9:4734

Yu Y, Fu J, Xu Y, Zhang J, Fei Ren F, Zhao H et al (2018) Genome resequencing reveals the evolutionary history of peach fruit edibility. Nat Commun 9:5404

Zapata L, Ding J, Willing E, Hartwig B, Bezdan D, Jiao W et al (2016) Chromosome-level assembly of Arabidopsis thaliana Ler reveals the extent of translocation and inversion polymorphisms. Proc Natl Acad Sci 113:4052-4060

Zhang Y, López-Girona E, Aranzana MJ (2018) molecular markers for flat fruit marker-assisted selection. Acta Hortic 1203: 79-84

Zhou S, Hu Z, Li F, Tian S, Zhu Z, Li A, Chen G (2019) Overexpression of SIOFP20 affects floral organ and pollen development. Hortic Res 6:125

Zhou H, Ma R, Gao L, Zhang J, Zhang A, Zhang X et al (2021) A 1.7-Mb chromosomal inversion downstream of a PpOFP1 gene is responsible for flat fruit shape in peach. Plant Biotechnol J 19:192-205

Zhu H, Chen PY, Zhong S, Dardick C, Callahan A, An YQ et al. (2020) Thermal-responsive genetic and epigenetic regulation of DAM cluster controlling dormancy and chilling requirement in peach floral buds. Hortic Res 7:114

Publisher's note Springer Nature remains neutral with regard to jurisdictional claims in published maps and institutional affiliations. 